PAPER

\title{
Incidence of amyotrophic lateral sclerosis in southern Italy: a population based study
}

\author{
G Logroscino, E Beghi, S Zoccolella, R Palagano, A Fraddosio, I L Simone, P Lamberti, V Lepore, \\ L Serlenga, the SLAP registry*
}

J Neurol Neurosurg Psychiatry 2005;76:1094-1098. doi: 10.1136/jnnp.2004.039180

See end of article for authors' affiliations

Correspondence to: Dr Giancarlo Logroscino, Department of Epidemiology HSPH 3819, Harvard University, 677 Huntington Avenue, Boston, MA 02115, USÁ; glogrosc@hsph.harvard. edu

*Members of the SLAP registry are listed in the appendix Received 10 February 2004 In revised form 21 November 2004 Accepted

25 November 2004

\begin{abstract}
Background: While the incidence of amyotrophic lateral sclerosis (ALS) is similar across the world (range, 1.0 to $2.5 / 100000$ ), a latitude gradient from north to south has been observed.

Objective: To determine the incidence of ALS in Puglia, a region of south eastern Italy, and to test the latitude gradient hypothesis comparing the present study with findings in studies conducted with the same design in a northern latitude.

Methods: Puglia (4086 613 residents in 2001) is the site of a multicentre-multisource prospective population based registry established in 1997. All incident ALS cases during the period 1998-99 were enrolled and followed up. Cases were classified using the first and the revised El Escorial criteria.

Results: During the study period 130 cases were enrolled. The annual crude incidence for ALS in Puglia for the two year period 1998-99 was 1.6/100 000 (95\% confidence interval, 1.3 to 1.9). The incidence was higher for men (incidence rate $(I R)=2.1(1.7$ to 2.7$)$ than for women $(I R=1.2(0.9$ to 1.5$)$ ) in all age groups, with a male to female ratio of 1.6. For both men and women, the incidence increased through age 75 and declined rapidly afterwards. The mean annual incidence adjusted by age and sex to the 2001 Italian population was $1.7 / 100000$ (1.4 to 2.0 ).

Conclusions: ALS incidence is within a narrow range across countries, with a peak between 65 and 75 years and a higher incidence in men. A north to south latitude gradient of ALS incidence is not supported by the results of cohort studies.
\end{abstract}

$\mathrm{E}$ pidemiological studies have shown that ALS is present with similar incidence in every part of the world. The incidence of ALS ranges between 1 and 2.5/100 000 in most studies, with the exception of former clusters in the western Pacific. ${ }^{1}$ Nevertheless, a latitude gradient from north to south has been suggested by a comparison of the incidence rates at different latitudes in the USA and Europe. ${ }^{2}$ It is not clear if the lower incidence rates reported in the Mediterranean area $^{3}{ }^{4}$ are real or reflect differences in case ascertainment, case definition, or time trends in the incidence of ALS, because these studies were conducted more than 20 years ago. Two recent European studies, one in Ireland and one in northern Italy, $^{56}$ reported similar incidences, using a prospective cohort design and the same case definition employing the first version of the El Escorial criteria. ${ }^{7}$

Our aim in this study was to determine the incidence of ALS in Puglia, a region of south eastern Italy, and to test the latitude gradient hypothesis comparing the present study with the findings of studies conducted with the same design and case definition in northern latitudes.

\section{METHODS}

\section{Study population}

This study was conducted in Puglia, a region in south eastern Italy with five administrative districts, called provinces. The area covers $19357 \mathrm{~km}^{2}$. The population of the study area was 4086613 , based on ISTAT data in 2001. This was the most accurate estimate of the reference population at the time of the study. There are 23 neurological units in the region of the survey and only one referral centre for ALS, based in the neurological department, University of Bari.

\section{Case ascertainment}

The main source of cases for this study is an ongoing multicentre prospective registry enrolling ALS incident cases, named SLAP (Sclerosi Laterale Amiotrofica - Puglia). The registry was established in 1997 and the case ascertainment began on 1 January 1998. The registry has several sources of information:

- The main source includes all neurological departments located in the area of the study. In each department one neurologist is in charge of recruitment of all newly diagnosed ALS patients for the registry. The neurologists at each centre use a standardised questionnaire with all clinical information necessary for the diagnosis and follow up. Demographics, laboratory findings, and diagnostic electromyography (EMG) are recorded as well. Each form is completed when patient's consent is obtained. This form is forwarded to the central unit of the registry. All the forms are reviewed by one of us (LS), who confirms the diagnosis and decides whether the patient is to be included in the registry. The forms are reviewed by the clinical committee only if there is a conflicting diagnosis between the site investigator and the clinical principal investigator. The final decision for these diagnoses is made on the clinical case records by the clinical committee of the study.

- AISLA, the lay Italian ALS association, cooperates with the registry.

Abbreviations: ALS, amyotrophic lateral sclerosis; PBP, progressive bulbar palsy; PLS, primary lateral sclerosis; PMA, progressive muscular atrophy; SLAP, Sclerosi Laterale Amiotrofica - Puglia 
- The registry is also linked to the hospital discharge diagnosis data bank (HDD-DB), a regional computerised system including the diagnosis of each hospital inpatient in Puglia for administrative purposes. At the end of each year the HDD-DB was searched for diagnosis of motor neurone disease (codes 335.2; International Classification of Diseases, 10 revision (ICD-10-CM)).

- After the introduction of riluzole as a licensed treatment for ALS in Italy in 1996, each patient taking riluzole needs to attend one of the outpatient clinics that distribute riluzole in the region of residency. Therefore it is highly probable that every ALS patient consulted a local neurologist at some point during the course of the disease.

The multisource system of case ascertainment allowed us to have a cross check confirmation of cases through different lists. Using this registry, we identified all residents 18 years of age or older in Puglia diagnosed with ALS in the two year period from 1 January 1998 to 31 December 1999. A subject was considered resident if he was living in the area during the 12 months preceding the onset of the disease.

\section{Diagnostic criteria}

The diagnosis of ALS was based on the first version of the El Escorial criteria. ${ }^{7}$ These criteria require the presence of signs in lower or upper motor neurones, or both, and progressive spread of the signs from one region to another, in the absence of neuroimaging and electrophysiological evidence of other diseases that might explain the clinical features. These criteria distinguish four levels of diagnostic certainty: definite, probable, possible, and suspected ALS. Individuals under the age of 18 were excluded to avoid misclassification of other motor neurone diseases of genetic origin, mimicking ALS. Cases with progressive bulbar palsy (PBP), progressive muscular atrophy (PMA), and primary lateral sclerosis (PLS) were included in the study and were reclassified according to the El Escorial criteria. ${ }^{7}$ We considered all the diagnostic levels for the calculation of incidence.

All cases that were included in this study had EMG. We used EMG to reclassify cases according to the revised ElEscorial criteria. ${ }^{8}$

\section{Statistical analysis}

The denominator for the calculation of the incident rates was the total resident population of Puglia in the year 2001. An incidence rate for the population older than 18 years was also calculated. Crude incidence rates were age and sex adjusted to the 2001 Italian population using direct standardisation. ${ }^{9}$

Confidence limits for incidence were calculated assuming a Poisson distribution. ${ }^{9}$ Rates were also calculated for each province. A standardised incidence ratio (SIR) was calculated using the ALS incidence in the entire region as the standard. ${ }^{10}$ Based on this we calculated the number of expected cases in each province and the ratio between observed and expected

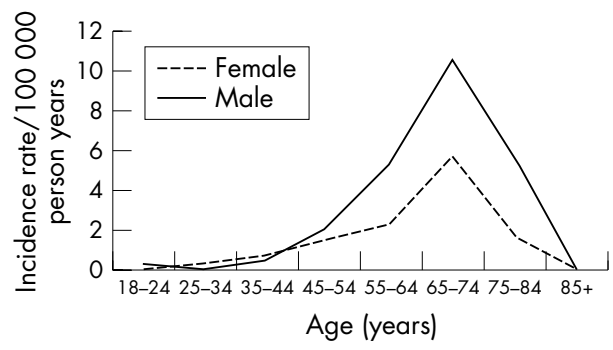

Figure 1 Change in incidence rate of amyotrophic lateral sclerosis with age. cases. Ninety five per cent confidence intervals (CI) of the SIR were also calculated.

Comparison with other studies was possible only through data on ages between 45 and 74 years. Standardised rates were calculated using the direct method for this age group with the 1990 US population as standard, because this was the standard used to make comparisons in previous studies. ${ }^{11}$

\section{RESULTS}

During the two year surveillance period, 130 patients meeting eligibility criteria were referred to the SLAP registry ( 81 male $(62.3 \%)$ and 49 female $(37.6 \%))$. Median age at diagnosis was 65.4 for men (range 19 to 80 years) and 64.2 for women (range 28 to 80 years). Ten patients were under 45 years old $(7.7 \%)$. All cases were reported by the primary sources of case ascertainment. There were only two familial ALS cases (1.5\%)

Of the 130 cases, there was one 53 year old man with PBP and with no sign of involvement of the second motor neurone. He was diagnosed three months after the onset of symptoms and became a definite case of ALS after six months of follow up. A 62 year old male patient presented with only upper motor neurone signs in three regions six months after the onset of symptoms. Follow up until the end of 2003 confirmed the lack of lower motor neurone involvement, and the diagnosis of PLS was made. There were 12 cases of PMA, nine male and three female, with a median age of 66.4 years and a median onset to diagnosis interval of 9.2 months. Six of 12 PMA cases became definite ALS cases during follow up, while two died within one year after the diagnosis with only lower motor neurone signs. PLS, PBP, and PMA were included in the study and were classified as suspected or possible ALS according to the first version of the El Escorial criteria. $^{7}$

The crude annual incidence rate for ALS in Puglia for the two year period 1998 to 1999 was 1.6/100 000 person years (95\% CI, 1.3 to 1.9$)$. The incidence rate was higher for men $(\mathrm{IR}=2.1(1.7$ to 2.6$))$ than for women $(\mathrm{IR}=1.2(0.9$ to 1.6$))$ in all age groups, with a male to female ratio of 1.6. This ratio was constant across all age groups. For both men and women, the incidence increased with age but declined markedly after age 75 (fig 1, table 1). The annual incidence, age and sex adjusted to the 2001 Italian population, was 1.7/ 100000 person years (95\% CI, 1.4 to 2.0 ).

The incidence rates of ALS in the five provinces are compared in table 2. The incidence rates ranged from 1.26 (Foggia) to 2.3 (Taranto) but the difference was not significant.

We compared our incidence rates with those from other studies for individuals between 45 and 74 years of age, adjusting all the rates to the US 1990 general population. The adjusted incidence rate for the 45 to 74 year age group was 4.1/100 000 person years (95\% CI, 3.4 to 5.0 ): 5.5 for men (95\% CI, 4.3 to 6.9 ) and 2.9 for women (95\% CI, 2.1 to 4.0 ) (table 3).

All cases that were included in this study had an EMG. We used EMG to reclassify cases according to the revised ElEscorial criteria. ${ }^{8}$ Of 45 possible cases, 29 (18 men and 11 women) were reclassified after EMG as probable laboratory supported ALS, while 16 were retained as clinically possible ALS (eight male, eight female). The pool of subjects available for ALS clinical trial drugs was 73 patients (definite + probable) using the first version of El Escorial criteria, and 102 using the revised El Escorial criteria.

\section{DISCUSSION}

Our study was conducted in a well defined geographical region in the Mediterranean area, using a prospective cohort design with a population based registry as the primary source of cases and El Escorial criteria for the diagnosis. ${ }^{78}$ These 
Table 1 Incidence rates of amyotrophic lateral sclerosis in men and women for Puglia, south east Italy (1998 to 1999)

\begin{tabular}{|c|c|c|c|c|c|c|c|c|}
\hline \multirow[b]{2}{*}{ Age (years) } & \multicolumn{4}{|l|}{ Male } & \multicolumn{4}{|l|}{ Female } \\
\hline & Population & Cases & Rate & $95 \% \mathrm{Cl}$ & Population & Cases & Rate & $95 \% \mathrm{Cl}$ \\
\hline 0 to 17 & 439604 & 0 & 0 & - & 413809 & 0 & 0 & - \\
\hline 18 to 24 & 215316 & 1 & 0.23 & 0.03 to 0.49 & 206887 & 0 & 0 & - \\
\hline 25 to 34 & 322393 & 0 & 0 & - & 324442 & 2 & 0.31 & 0.07 to 1.24 \\
\hline 35 to 44 & 288315 & 3 & 0.5 & 0.16 to 1.6 & 300169 & 4 & 0.67 & 0.25 to 1.78 \\
\hline 45 to 54 & 245347 & 11 & 2.24 & 1.81 to 4.04 & 266441 & 8 & 1.5 & 0.75 to 3.0 \\
\hline 55 to 64 & 203593 & 22 & 5.4 & 3.55 to 8.20 & 220803 & 10 & 2.26 & 1.22 to 1.43 \\
\hline 65 to 74 & 164781 & 35 & 10.6 & 7.61 to 14.76 & 201017 & 21 & 5.22 & 3.40 to 8.01 \\
\hline 75 to 84 & 79262 & 9 & 5.67 & 2.95 to 10.90 & 118917 & 4 & 1.68 & 0.63 to 4.48 \\
\hline $85+$ & 22282 & 0 & 0 & - & 44230 & 0 & 0 & - \\
\hline Crude IR & 1989893 & 81 & 2.1 & 1.69 to 2.61 & 2096715 & 49 & 1.17 & 0.88 to 1.57 \\
\hline
\end{tabular}

Among individuals over 18 years, the average incidence rate was $2.6(95 \% \mathrm{Cl}, 2.1$ to 3.2$)$ in men and $1.5(0.9$ to 2.6$)$ in women.

$\mathrm{Cl}$, confidence interval; IR, incidence rate.

features allowed us to compare the results of this study with other studies conducted using the same methodology in the USA and Europe. Four studies ${ }^{5}{ }^{1213}$ have been published with a prospective design and with data collected between 1989 and 1996, and two of these ${ }^{56}$ used identical criteria for the classification of cases. ${ }^{7}$

The incidence of ALS in Puglia, south eastern Italy, was slightly lower than reported in other studies conducted over the same period with the same case definition and the same design. The results of this study, conducted in a relatively small geographical area, confirm that the ALS incidence is within a narrow range across several countries when a comparison with previous European studies is made (table 3 ). A north to south latitude gradient does not explain the small variation of ALS incidence in Europe.

The incidence was higher for men than for women, as in previous studies. This sex difference could reflect underascertainment of ALS among women. This is unlikely because the age incidence curves of the two sexes are of similar shape (fig 1), and the interval between the onset of symptoms and diagnosis was ALS similar in men and women (about nine months). This would be unlikely if the referral process for ALS was different for men and women. An alternative explanation is that the difference in incidence is real. The male to female ratio in this study was lower than in previous studies in the Mediterranean areas: in Sardinia ${ }^{4}$ it was 2.6 to 1 and in Israel, 1.8 to $1 .{ }^{3}$ These studies were conducted in the late seventies and early eighties. It is possible that in the last 20 years the incidence of ALS has increased more in men than in women in countries at a southern latitude. Women may be less exposed to some environmental risk factor than men in the Mediterranean countries. One possible risk factor that could have contributed to this sex difference is smoking. Smoking has been reported as a risk factor for ALS, with a dose-response effect, in two case-control studies, ${ }^{14}{ }^{15}$ and women are less likely to smoke than men in the elderly age range. ${ }^{16}$

We observed an increase in the age specific incidence in both sexes, with a peak between 65 and 75 years of age. All the most recent population based studies show an increase in the median age of onset compared with previous studies: 63.7 years in Piemonte, northern Italy; 63.3 years in Ireland; 64.6 in this series from Puglia, southern Italy. ${ }^{56}$ The annual incidence for ALS was consistently higher for men than for women in all age groups, with the greatest difference noted in the early seventies. Only in Rochester (Minnesota, USA) has a constant increase with age been reported previously. ${ }^{17} \mathrm{~A}$ more recent study from the same area in a different time period (1990 to 1998) showed that the incidence peaks in the sixties and then there is a rapid decline, consistent with the findings from other studies. ${ }^{18}$ The presence of a specific peak in incidence could indicate that the ALS onset is at least partly independent of other aging related processes. The presence of an age peak may indicate the existence of a specific induction period of the disease which depends on the susceptibility to particular risk factors. A specific time of exposure or specific dose of one or more environmental risk factors could be necessary for the onset of disease.

The decline in the older age groups might also reflect underascertainment, as previously suggested. ${ }^{19}$ The diagnosis may be difficult because of the presence of other diseases affecting the bones, joints, and nervous system. It is less likely that a patient over 80 years of age would be referred to a neurologist than a younger person. Even if an older person is referred to a neurologist, the shorter life expectancy in that age group could result in a less aggressive search for the ALS diagnosis. A door to door population based study might unravel the issue of the decline in ALS incidence in older age groups, but such studies are extremely difficult to conduct because of the low incidence of ALS even among the elderly.

Table 2 Crude and age and sex-adjusted incidence mean annual rate by province of residence

\begin{tabular}{lllllll}
\hline Province & Population & Observed cases & Crude IR/10 & Expected cases* & $\begin{array}{l}\text { Standardised incidence } \\
\text { ratio }\end{array}$ & $95 \% \mathrm{Cl}$ \\
\hline Bari & 1560520 & 47 & 1.51 & 49.6 & 0.95 & 0.7 to 1.2 \\
Foggia & 697402 & 17 & 1.22 & 22.2 & 0.77 & 0.4 to 1.1 \\
Taranto & 586972 & 27 & 2.30 & 18.7 & 1.44 & 0.9 to 3.0 \\
Brindisi & 411051 & 11 & 1.34 & 13.1 & 0.84 & 0.4 to 1.4 \\
Lecce & 815685 & 28 & 1.71 & 25.9 & 1.08 & 0.7 to 1.4 \\
Puglia & 4086761 & 130 & 1.59 & & 1.00 & \\
\hline
\end{tabular}

*Based on the crude incidence rate of Puglia.

$\mathrm{Cl}$, confidence interval; IR, incidence rate. 
Table 3 Incidence of ALS in men and women 45 to 74 years old by latitude gradient in selected studies in Europe which used a prospective cohort design, age adjusted to the 1990 US population

\begin{tabular}{|c|c|c|c|c|c|c|}
\hline Country & Years & Latitude $\left({ }^{\circ}\right)$ & IR men & IR women & Combined IR & $95 \% \mathrm{Cl} \mathrm{IR}$ \\
\hline Scotland & 1989 & 55 to 59 & 6.7 & 3.8 & 5.2 & 4.1 to 6.4 \\
\hline Ireland & 1995 to 1997 & 52 to 55 & 6.7 & 5.3 & 6.0 & 5.1 to 6.9 \\
\hline Piemonte (Italy) & 1995 to 1996 & 44 to 46 & 5.9 & 4.7 & 5.4 & 4.6 to 6.3 \\
\hline Puglia (Italy) & 1998 to 1999 & 40 to 42 & 5.5 & 2.9 & 4.1 & 2.6 to 5.7 \\
\hline
\end{tabular}

We included all four diagnostic levels of the first version of El Escorial, including PLS and PMA, in the calculation of the incidence rates. These diagnostic categories were included in the Scottish and the Irish studies but not in the one conducted in Piemonte. ${ }^{5612}$ The number of cases of PMA was particularly high in our series-about eight times higher than reported in the Irish study. Two individuals died without signs of upper neurone involvement and six became definite ALS. There are several pieces of evidence showing that most patients with PMA (suspect ALS) evolve to typical ALS. About $10 \%$ of those who die from ALS are still classified as suspected or possible according to the El Escorial criteria. ${ }^{20}$ The involvement of the pyramidal tracts has been shown in necropsy series of PMA or suspect ALS cases. ${ }^{21}$ Thus the identification of cases of suspected ALS and their inclusion in ALS epidemiological studies is important for describing the whole spectrum of this clinically heterogeneous disease.

In this population based setting, the use of the revised El Escorial criteria ${ }^{8}$ increased the pool of ALS patients available for clinical trials by almost $30 \%$. We did not observe a significant regional variation in incidence. Regional variations were found in some of the previous studies ${ }^{5}$ but not in others. ${ }^{6}{ }^{12}$

The main strength of our study is that complete case ascertainment is highly probable owing to the multisource system used for the identification of the ALS cases from a stable reference population living in a well defined geographical area. Nevertheless, some atypical or very elderly cases who either did not receive any medical attention or were improperly diagnosed could have been missed.

\section{Conclusions}

This study, conducted in the Mediterranean region, supports previous work suggesting that ALS is age related, commoner in men, and has the same incidence in different geographical areas. The even incidence rates across populations opposes the view that environmental risk factors play a major role in causation of ALS. An alternative explanation is that diffuse environmental risk factors for ALS are present homogeneously at different latitudes in Europe. In a population based setting, both case-control and cohort studies are often unable to detect risk factors with insufficient heterogeneity because of lack of power. ${ }^{22}$ If this is the case, to further clarify the role of environmental factors, a possible strategy could be to conduct collaborative analytical studies with incident cases. A collaborative project of population based ALS registries (EURALS) is being implemented in Europe and will provide the basis for this type of study.

\section{ACKNOWLEDGEMENTS}

We own a particular thank to Mr Camillo Colapinto who was the first to launch the idea of a regional registry for ALS in Puglia and always gave us a great support of enthusiasm and new ideas both in person and through the AILSA.

\section{Authors' affiliations}

G Logroscino, Department of Epidemiology HSPH, Harvard University, Boston, Massachusetts, USA
E Beghi, Istituto Ricerche Farmacologiche Mario Negri, Milan, Italy S Zoccolella, R Palagano, A Fraddosio, I L Simone, P Lamberti, V Lepore, Department of Neurological Sciences, University of Bari, Bari, Italy

L Serlenga, Operative Unit of Neurology, Andria (BA), Italy

Competing interests: none declared

\section{APPENDIX}

Sclerosi Laterale Amiotrofica - Puglia (SLAP Registry) Principal investigators: Giancarlo Logroscino ( Boston); Luigi Selenga (Andria)

Scientific committee: Ettore Beghi, Vito Lepore, Paolo Livrea, Giancarlo Logroscino, Isabella L. Simone, Luigi Serlenga.

Clinical committee: Paolo Lamberti, Bruno Maggio, Bruno Passarella, Vito Santamato, Luigi Serlenga, Isabella Simone, Pasquale Simone, Franco Valluzzi.

Epidemiologic and Data Management Unit: Vito Lepore, Saverio Staffieri.

Study monitors: Angela Fraddosio, Rino Palagano, Stefano Zocolella.

SLAP neurologists: Giuseppe Belfiore ( Lecce), Giuseppe Benedetto (Noci), Nicola Cacudi (S Paolo, Bari), Antonio Cazzato (Lecce), Pasquale Colamartino (Bisceglie), Pietro Di Viesti (S Giovanni Rotondo), Silvana Epifani (Galatina) Francesco Lincesso (Taranto) Bruno Maggio (Conversano), Vincenzo Monitillo (Cassano Murge), Angelo Moramarco (Altamura), Antonello Nicolaci (Scorrano), Cecilia Nozzoli (Brindisi), Sergio Pasca (Casarano), Rosaria Pulimeno (Gallipoli), Giuseppe Russo (Grottaglie), Vito Santamato (DiVenere, Bari), Isabella Laura Simone (Policlinico, Bari), Giovanni Strafella (Andria), Maria Terraciano (Foggia), Paolo Tota (Barletta), Francesco Valluzzi (Putignano).

SLAP writing group: G Logroscino, E Beghi, S Zoccolella, $\mathrm{R}$ Palagano, A Fraddosio, I L Simone, P Lamberti, V Lepore, and L Serlenga.

\section{REFERENCES}

1 Roman GC. Neuroepidemiology of amyotrophic lateral sclerosis: clues to aetiology and pathogenesis. J Neurol Neurosurg Psychiatry 1996;61:131-7.

2 Annegers JF, Appel S, Lee JRJ, et al. Incidence and prevalence of amyotrophic lateral sclerosis in Harris County, Texas, 1985-1988. Arch Neurol 1991;48:589-93.

3 Kahana E, N Z. Changes in the incidence of amyotrophic lateral sclerosis in Israel. Arch Neurol 1984;41:157-60.

4 Rosati G, Pinna L, Granieri E, et al. Studies on epidemiological, clinical, and etiological aspects of ALS disease in Sardinia, Southern Italy. Acta Neurol Scand 1977;55:231-44.

5 Traynor BJ, Codd MB, Corr B, et al. Incidence and prevalence of ALS in Ireland, 1995-1997: a population-based study. Neurology 1999:52:504-9.

6 Piemonte, Valle d'Aosta Register for Amyotrophic Lateral Sclerosis (PARALS). Incidence of ALS in Italy. Evidence for a uniform frequency in Western countries. Neurology 2001;56:239-44.

7 Brooks BR. El Escorial World Federation of Neurology criteria for the diagnosis of amyotrophic lateral sclerosis. Subcommittee on Motor Neuron Diseases/Amyotrophic Lateral Sclerosis of the World Federation of Neurology Research Group on Neuromuscular Diseases and the El Escorial "Clinical limits of amyotrophic lateral sclerosis" workshop contributors. J Neurol Sci 1994:124(suppl):96-107.

8 Brooks BR, Miller RG, Swash M, et al. El Escorial revisited: revised criteria for the diagnosis of amyotrophic lateral sclerosis. Amyotroph Lateral Scler Other Motor Neuron Disord 2000;1:293-9. 
9 Woodward M. Epidemiology: study design and data analysis. Boca Raton: Chapman and Hall/CRC Press, 1999:159-67.

10 Statistica IId. demo.istat.it/popl/start.html. Istituo Italiano di Statistica [web site]. Available at: demo.istat.it/popl/start.html.

11 Census Bureau US. 1990 Census. Available at: http://eire.census.gov/ popest/archives/national.

12 Swingler RJ, Fraser H, Warlow CP. Motor neuron disease and polio in Scotland. J Neurol Neurosurg Psychiatry 1992;55:1116-20.

13 McGuire V, Longstreth WT, Koepsell TD, et al. Incidence of amyotrophic lateral sclerosis in three counties in western Washington state. Neurology 1996:47:571-3.

14 Kamel F, Umbach D, Munsat T, et al. Association of cigarette smoking with amyotrophic lateral sclerosis. Neuroepidemiology 1999; 18:194-202.

15 Nelson LM, McGuire V, Longstreth WT, et al. Population-based casecontrol study of amyotrophic lateral sclerosis in western Washington state. I. Cigarette smoking and alcohol consumption. Am J Epidemiol 2000;151:156-63.
16 Schiaffino A, Fernandez E, Borrell C, et al. Gender and educational differences in smoking initiation rates in Spain from 1948 to 1992. Eur J Public Health 2003;13:56-60.

17 Yoshida S, Mulder DW, Kurland LT, et al. Follow-up study of amyotrophic lateral sclerosis in Rochester, Minn, 1925 through 1984. Neuroepidemiology 1986:5:61-70

18 Sorenson EJ, Stalker AP, Kurland LT, et al. Amyotrophic lateral sclerosis in Olmsted County, Minnesota, 1925 to 1998. Neurology 2002;59:280-2.

19 Chancellor AM, Warlow CP. Adult onset motor neuron disease: worldwide mortality, incidence and distribution since 1950. J Neurol Neurosurg Psychiatry 1992;55:1106-15.

20 Traynor BJ, Codd MB, Corr B, et al. Clinical features of amyotrophic latera sclerosis according to the El Escorial and Airlie House diagnostic criteria: a population-based study. Arch Neurol 2000;57:1171-6.

21 Ince PG, Evans J, Knopp M, et al. Corticospinal tract degeneration in the progressive muscular atrophy variant of ALS. Neurology 2003;60:1252-8.

22 Rose G. Sick individuals and sick populations. Int J Epidemiol 1985;14:32-8.

\section{Clinical Evidence - Call for contributors}

Clinical Evidence is a regularly updated evidence-based journal available worldwide both as a paper version and on the internet. Clinical Evidence needs to recruit a number of new contributors. Contributors are healthcare professionals or epidemiologists with experience in evidence-based medicine and the ability to write in a concise and structured way.

Areas for which we are currently seeking authors:

- Child health: nocturnal enuresis

- Eye disorders: bacterial conjunctivitis

- Male health: prostate cancer (metastatic)

- Women's health: pre-menstrual syndrome; pyelonephritis in non-pregnant women

However, we are always looking for others, so do not let this list discourage you.

Being a contributor involves:

- Selecting from a validated, screened search (performed by in-house Information Specialists) epidemiologically sound studies for inclusion.

- Documenting your decisions about which studies to include on an inclusion and exclusion form, which we keep on file.

- Writing the text to a highly structured template (about 1500-3000 words), using evidence from the final studies chosen, within 8-10 weeks of receiving the literature search.

- Working with Clinical Evidence editors to ensure that the final text meets epidemiological and style standards.

- Updating the text every six months using any new, sound evidence that becomes available. The Clinical Evidence in-house team will conduct the searches for contributors; your task is simply to filter out high quality studies and incorporate them in the existing text.

- To expand the topic to include a new question about once every 12-18 months.

If you would like to become a contributor for Clinical Evidence or require more information about what this involves please send your contact details and a copy of your CV, clearly stating the clinical area you are interested in, to Klara Brunnhuber (kbrunnhuber@ bmigroup.com).

\section{Call for peer reviewers}

Clinical Evidence also needs to recruit a number of new peer reviewers specifically with an interest in the clinical areas stated above, and also others related to general practice. Peer reviewers are healthcare professionals or epidemiologists with experience in evidence-based medicine. As a peer reviewer you would be asked for your views on the clinical relevance, validity, and accessibility of specific topics within the journal, and their usefulness to the intended audience (international generalists and healthcare professionals, possibly with limited statistical knowledge). Topics are usually 1500-3000 words in length and we would ask you to review between 2-5 topics per year. The peer review process takes place throughout the year, and our turnaround time for each review is ideally 10-14 days.

If you are interested in becoming a peer reviewer for Clinical Evidence, please complete the peer review questionnaire at www.clinicalevidence.com or contact Klara Brunnhuber (kbrunnhuber@bmigroup.com). 